Cahiers $d u$ MONDE RUSSE

\section{Cahiers du monde russe}

Russie - Empire russe - Union soviétique et États indépendants

$43 / 4 \mid 2002$

Intellectuels et intelligentsia

\title{
Andrei V. Golovnev, Gail Oshrenko, Siberian survival | Marjorie Mandelstam Balzer, The tenacity of ethnicity
}

\section{Elisabeth Gessat-Anstett}

\section{(2) OpenEdition}

Édition électronique

URL : https://journals.openedition.org/monderusse/4052

DOI : $10.4000 /$ monderusse. 4052

ISSN : $1777-5388$

Éditeur

Éditions de l'EHESS

Édition imprimée

Date de publication : 30 décembre 2002

Pagination : 782-784

ISBN : 2-7132-1796-2

ISSN : $1252-6576$

Référence électronique

Elisabeth Gessat-Anstett, « Andrei V. Golovnev, Gail Oshrenko, Siberian survival | Marjorie

Mandelstam Balzer, The tenacity of ethnicity », Cahiers du monde russe [En ligne], 43/4 | 2002, mis en ligne le 03 juillet 2009, consulté le 03 septembre 2022. URL : http://journals.openedition.org/ monderusse/4052 ; DOI : https://doi.org/10.4000/monderusse.4052

Ce document a été généré automatiquement le 3 septembre 2022.

Tous droits réservés 


\title{
Andrei V. Golovnev, Gail Oshrenko, Siberian survival | Marjorie Mandelstam Balzer, The tenacity of ethnicity
}

\author{
Elisabeth Gessat-Anstett
}

\section{RÉFÉRENCE}

Andrei V. GOLOVNEV, Gail OSHRENKO, Siberian survival. The Nenets and their story, Ithaca-Londres, Cornell University Press, 1999, 176 p.

Marjorie MANDELSTAM BALZER, The tenacity of ethnicity. Siberian saga in global

perspective, Princeton, NJ, Princeton University Press, 1999, 326 p.

1 Les deux textes recensés offrent un bon exemple de la façon dont l'anthropologie sociale américaine approche l'étude des minorités ethniques de l'espace sibérien postsoviétique.

2 L'ouvrage de Andrei Golovnev et Gail Oshrenko, tout d'abord, est le fruit d'une collaboration entre un ethnologue russe et une juriste américaine. Il est consacré à l'étude d'une communauté Nénetse de la péninsule de Yamal au nord-ouest de la Sibérie, adoptant un mode de vie semi-nomade fondé sur l'élevage du renne. Cet ouvrage s'appuie sur trois courtes enquêtes de terrain réalisées durant les étés 1992, 1993 et 1994, et autant d'années de recherches, qui visaient à faire le bilan des changements institutionnels, sociaux et culturels intervenus au sein de cette communauté indigène de Russie durant les années de glasnost'.

3 L'ouvrage de Marjorie Mandelstam Balzer est, quant à lui, consacré aux Kanthes, des communautés du centre de la Sibérie occidentale. Entamée dès les années 1970 sur la base d'un intérêt pour l'histoire des peuples indigènes soviétiques, et concrétisée dès cette époque par plusieurs séjours sur le terrain, l'étude de M. Mandelstam Balzer s'est 
poursuivie et achevée au cours des années 1990, par le biais d'échanges scientifiques renouvelés avec diverses institutions russes de recherche qui lui ont donné accès à de nouveaux matériaux, ethnographiques et archivistiques. Le propos vise, là encore, à présenter un bilan des transformations socio-culturelles intervenues au sein de ces communautés nomades de Sibérie.

4 Ces deux ouvrages comportent de très nombreux points communs. Le premier est de choisir un fil chronologique et de présenter une histoire critique des communautés étudiées en prenant pour point de départ un analogue étonnement pour la pérennité identitaire dont elles ont pu faire preuve dans une conjoncture d'ensemble peu favorable au maintien de traditions et de survivances culturelles particulières.

5 Les deux textes privilégient ainsi la restitution des enjeux politiques, économiques et juridiques qui ont marqué la vie des communautés étudiées et qui ont contraint ces dernières à négocier les voies de leur perpétuation. Ils s'appuient de façon forte sur la prise en compte de l'aspect matériel du mode de vie, à partir d'un contraste marqué entre ce qui relèverait d'une tradition culturelle à l'intérieur de la communauté, et ce qui relèverait d'une modernité acculturée à l'extérieur. Il est par ailleurs curieux de remarquer que les auteurs utilisent en illustration les mêmes pictogrammes de rennes pour ponctuer les têtes de chapitre ou encore la page de couverture et de dédicace.

6 L'ouvrage de A.Golovnev et G. Oshrenko comme celui de M. Mandelstam Balzer prennent en outre le parti de se focaliser sur une opposition entre le global (relevant des espaces russes, soviétiques, mondiaux) et le local qui fait à chaque fois référence à la communauté étudiée. Et ils concluent de façon similaire sur le futur incertain de ces communautés et sur les nécessaires aménagements, ruptures ou résistances dont les Kanthes aussi bien que les Nénetses auront à faire preuve.

7 L'intérêt commun à ces deux ouvrages est d'offrir une vision d'ensemble du passé et du présent de ces communautés en donnant une lecture analytique de leur situation, relevant d'une sorte de géopolitique culturelle. Ils permettent en effet de situer ces deux petites sociétés sibériennes dans leur environnement géographique et socio-historique et de comprendre les problèmes que ces communautés affrontent lorsqu'il s'agit de l'enjeu stratégique de leur autonomie politique, économique et culturelle.

8 Le maintien d'une identité communautaire demeure en effet, ici comme partout ailleurs, lié à la possibilité de contrôler et d'aménager un territoire, et s'oppose donc souvent aux intérêts nationaux ou internationaux de la Fédération de Russie. On doit ainsi porter au crédit de ces deux textes de mettre pleinement en lumière l'importance de l'espace et de son usage dans la pérennité culturelle et démographique de groupes sociaux spécifiques.

9 Les deux ouvrages divergent principalement sur la portée des analyses et l'ampleur des matériaux présentés. A. Golovnev et G. Oshrenko nous présentent en effet un texte court qui effectue de façon assez succincte la synthèse de sources souvent disparates à partir d'une simple juxtaposition de matériaux et de points de vue (historiques, économiques, juridiques, religieux). Cependant que l'étude de M. Mandelstam Balzer s'appuie sur un long travail d'enquête, poursuivi - malgré des interruptions - sur plusieurs dizaines d'années et qui révèle, tout au long du texte et de l'important appareil de notes qui lui succède, sa finesse et sa richesse. Attentive à la restitution du propos commenté des acteurs sociaux, M. Mandelstam Balzer montre avec précision la complexité et la portée identitaire forte de certaines pratiques, notamment religieuses. Elle s'attache également à reconstituer les racines des grandes théories de l'ethnicité telles qu'elles peuvent être théorisées et utilisées 
par certaines doctrines ethno-nationalistes sibériennes en pointant les jeux complexes introduits entre des logiques politiques de tribalisation et de globalisation.

Il n'en reste pas moins que la tonalité ethnographique des ouvrages ne masque pas toujours la fragilité du discours proprement anthropologique. Le lecteur regrettera ainsi l'absence d'une dimension comparative avec la société allochtone -i.e. l'espace intellectuel, idéologique et culturel de la Russie soviétique et post-soviétique - ou avec d'autres communautés sibériennes. Car les traits sociaux ou les situations présentées comme spécifiques de l'évolution de ces micro-sociétés renvoient trop souvent à une réalité autrefois déjà très répandue dans l'espace soviétique (importance de l'alcoolisme et du suicide, acculturation croissante des jeunes générations), et se rapportent de façon plus générale au dépérissement de l'espace rural et à la décomposition sociale des communautés urbaines post-soviétiques. Comparées à d'autres matériaux du même ordre collectés ailleurs, ces observations permettraient de restituer la véritable originalité de ces groupes sociaux, sans la réduire à une simple survivance d'archaïsmes technologiques ou de croyances religieuses primitives.

Une perception de l'intérieur de ces petites communautés, de la complexité de leur organisation et de leur perpétuation sociale fait ainsi défaut. Une absence qui apporte une certaine gêne dans la compréhension extensive des questions traitées, et qui est peut-être due à la façon (rapide pour A. Golovnev et G. Oshrenko, fragmentée dans le temps pour M. Mandelstam Balzer) dont les données ethnographiques de terrain purent être collectées.

Par-delà une description du contexte déterminant de l'espace social et culturel ambiant, russifié, soviétisé, et dans une certaine mesure en cours de désoviétisation, manquent ainsi la restitution des repères, des valeurs et des représentations fondamentalement propres aux communautés étudiées, de même qu'une présentation de la façon dont des éléments de diverses natures et origines (espace russe ou soviétique et espace indigène, culture matérielle et culture idéelle) s'assemblent pour produire un système symbolique cohérent, seul à même d'assurer la pérennité identitaire de ces micro-sociétés.

Car s'il est évident que les communautés observées n'échappent pas au monde, c'est singulièrement ignorer leur spécificité que d'oublier leur propre mode de représentation d'une réalité qu'ils sont les seuls à vivre. Une réalité que leurs observateurs envisagent certes de façon globale, sans parvenir à nous convaincre tout à fait que les populations autochtones de la Sibérie post-soviétique perçoivent et intègrent absolument ce point de vue. 